\title{
Penetration of echinocandins into wound secretion of critically ill patients
}

\author{
Tiziana Gasperetti ${ }^{1} \cdot$ René Welte $^{1} \cdot$ Herbert Oberacher $^{2} \cdot$ Jana Marx ${ }^{1} \cdot$ Ingo Lorenz $^{3} \cdot$ Peter Schellongowski ${ }^{4}$. \\ Thomas Staudinger ${ }^{4} \cdot$ Karin Burgmann $^{4} \cdot$ Philipp Eller $^{5} \cdot$ Tobias Santner $^{6} \cdot$ Andrea Griesmacher $^{6} \cdot$ Hartwig Pfisterer $^{6}$. \\ Stephan Eschertzhuber ${ }^{7,10} \cdot$ Maria Aigner $^{8,11} \cdot$ Michael Joannidis $^{9} \cdot$ Romuald Bellmann $^{1}$ (D)
}

Received: 22 December 2020 / Accepted: 8 March 2021 / Published online: 20 April 2021

(c) The Author(s) 2021

\begin{abstract}
Purpose Wound infections caused by Candida are life-threatening and difficult to treat. Echinocandins are highly effective against Candida species and recommended for treatment of invasive candidiasis. As penetration of echinocandins into wounds is largely unknown, we measured the concentrations of the echinocandins anidulafungin (AFG), micafungin (MFG), and caspofungin (CAS) in wound secretion (WS) and in plasma of critically ill patients.

Methods We included critically ill adults with an indwelling wound drainage or undergoing vacuum-assisted closure therapy, who were treated with an echinocandin for suspected or proven invasive fungal infection. Concentrations were measured by liquid chromatography with UV (AFG and MFG) or tandem mass spectrometry detection (CAS).

Results Twenty-one patients were enrolled. From eight patients, serial WS samples and simultaneous plasma samples were obtained within a dosage interval. AFG concentrations in WS amounted to $<0.025-2.25 \mathrm{mg} / \mathrm{L}$, MFG concentrations were $0.025-2.53 \mathrm{mg} / \mathrm{L}$, and CAS achieved concentrations of 0.18-4.04 mg/L. Concentrations in WS were significantly lower than the simultaneous plasma concentrations and below the MIC values of some relevant pathogens.

Conclusion Echinocandin penetration into WS displays a high inter-individual variability. In WS of some of the patients, concentrations may be sub-therapeutic. However, the relevance of sub-therapeutic concentrations is unknown as no correlation has been established between concentration data and clinical outcome. Nevertheless, in the absence of clinical outcome studies, our data do not support the use of echinocandins at standard doses for the treatment of fungal wound infections, but underline the pivotal role of surgical debridement.
\end{abstract}

Keywords Echinocandin antifungals $\cdot$ Target-site pharmacokinetics $\cdot$ Wound infection $\cdot$ Invasive candidiasis $\cdot$ Vacuum assisted closure therapy

Romuald Bellmann

romuald.bellmann@i-med.ac.at

1 Clinical Pharmacokinetics Unit, Division of Intensive Care and Emergency Medicine, Department of Internal Medicine I, Medical University of Innsbruck, Anichstrasse 35, 6020 Innsbruck, Austria

2 Institute of Legal Medicine and Core Facility Metabolomics, Medical University of Innsbruck, Innsbruck, Austria

3 General and Surgical ICU, University Hospital for Anaesthesia and Intensive Care, Medical University of Innsbruck, Innsbruck, Austria

4 Intensive Care Unit, Department of Internal Medicine I, Medical University of Vienna, Vienna, Austria

5 Intensive Care Unit, Department of Internal Medicine, University Hospital of Graz, Graz, Austria
6 Central Institute for Medical and Chemical Laboratory Diagnostics, Innsbruck General Hospital, Innsbruck, Austria

7 Transplant ICU, University Hospital for Anaesthesia and Intensive Care, Innsbruck General Hospital and Innsbruck Medical University Innsbruck, Innsbruck, Austria

8 Institute of Hygiene and Medical Microbiology, Medical University of Innsbruck, Innsbruck, Austria

9 Division of Intensive Care and Emergency Medicine, Department of Internal Medicine I, Medical University of Innsbruck, Innsbruck, Austria

10 Present Address: Department of Anaesthesia and Critical Care, District Hospital of Hall in Tyrol, Hall in Tyrol, Austria

11 Present Address: INNPATH GmbH, Tirol Kliniken, Innsbruck, Austria 


\section{Introduction}

Wound infections by Candida occur mainly after surgery, severe burns or traumatic injuries [1-3]. The outcome of this devastating and life-threatening condition largely depends on surgical debridement and on appropriate antifungal treatment [4]. However, the optimal antifungal drug regimen for Candida wound infections remains to be established [5]. Echinocandins are cyclic hexapeptides, which are highly effective against most of the pathogenic Candida species. Based on their efficacy against candidemia, echinocandins are recommended for the treatment of invasive candidiasis by current guidelines [6]. However, there is only one report on wound penetration of an echinocandin [7]. Therefore, we measured the concentrations of the commercially available echinocandins anidulafungin (AFG), micafungin (MFG), and caspofungin (CAS) in wound secretion (WS) and in simultaneously drawn plasma samples of critically ill patients treated with AFG, MFG, or CAS for suspected or proven invasive fungal infection.

\section{Study population and methods}

\section{Study design and patient enrolment}

This was an open-label, pharmacokinetic multi-centre study. The protocol was approved by the local ethics committees (EudraCT no. 2013-005065-38), and the study was carried out in accordance with the Declaration of Helsinki and with Austrian law. Written informed consent was obtained from competent patients, post-hoc consent from patients who were incompetent at the time of enrolment.

Consecutive critically ill adults were eligible if they fulfilled the following inclusion criteria: (1) ongoing echinocandin treatment with either AFG, MFG, or CAS for suspected or proven invasive candidiasis, and (2) indwelling wound drainage at any anatomical site or ongoing vacuumassisted closure (V.A.C.) therapy.

\section{Echinocandin treatment}

AFG (Ecalta®; Pfizer, Sandwich, Kent, UK), MFG (Mycamine ${ }^{\circledR}$; Astellas, Leiderdorp, NL), and CAS (Cancidas ${ }^{\circledR}$; Merck Sharp and Dohme, Hoddesdon, Hertfordshire, UK), respectively, were administered at the discretion of the treating physician. As recommended by the manufacturer, the daily maintenance dose of AFG amounted to $100 \mathrm{mg}$ after a 200-mg loading dose. MFG was given at a daily dose of $100 \mathrm{mg}$. The standard maintenance dose of CAS is $50 \mathrm{mg}$ daily after a single-loading dose of $70 \mathrm{mg}$. Patients with a body weight above $80 \mathrm{~kg}$ should receive $70 \mathrm{mg}$ daily during the entire treatment.

\section{Sampling and echinocandin quantification}

Samples were taken after the first echinocandin dose or after multiple doses in accordance with clinical requirements and the availability of WS. When WS was continuously drained into collection bags, a highly variable delivery of WS had to be considered. Whenever sufficient amounts of WS were yielded within a dosage interval, the bags were changed before the echinocandin infusion, as well as at 1, 4, 8, 12, 18 , and $24 \mathrm{~h}$ after the start of infusion, and kept for analysis. When delivery of WS was insufficient for serial sampling $(<0.5 \mathrm{~mL})$, the collection bag was changed only once or twice, and taken for echinocandin quantification. In the case of incomplete serial sampling on the first study day due to poor WS delivery, additional samples were taken the following day, if available. From patients with two or three indwelling wound drainages, WS was sampled simultaneously from these drains, if available. In patients undergoing V.A.C. therapy, the change of the V.A.C. container was scheduled according to the clinical requirements and kept for echinocandin quantification. Simultaneously with the change of the collection bags or V.A.C. containers, $2 \mathrm{~mL}$ of blood was drawn from the arterial line using heparinized vials (Sarstedt, Nümbrecht, Germany). Whole blood was centrifuged at $350 \times g$ for $10 \mathrm{~min}$ to obtain plasma. WS and plasma were stored at $-80{ }^{\circ} \mathrm{C}$ until analysis. As detailed in Online Resource 1, AFG and MFG were quantified by high-performance liquid chromatography with UV detection [8]; whereas, CAS concentrations were measured by means of liquid chromatography-tandem mass spectrometry (LC-MS/MS).

\section{Data analysis}

Echinocandin pharmacokinetics were calculated by a noncompartmental model using Kinetica $2000 \circledR$ (InnaPhase Corporation, Champs-sur-Marne, France). The area under the concentration-time curve from the start of the echinocandin infusion to the last sampling $\left(\mathrm{AUC}_{0-\mathrm{n}}\right)$ was computed using the log-linear method when the concentration in a trapezoid decreased, or with the trapezoidal method if the concentration increased. When serial WS samples had been obtained within the dosage interval, the penetration ratio (PR) was defined as the ratio between the $\mathrm{AUC}_{0-\mathrm{n}}$ over the same sampling period in $\mathrm{WS}$ and in plasma $\left(\mathrm{AUC}_{0 \text {-n }}\right.$ ${ }_{\text {ws }} / \mathrm{AUC}_{0 \text {-n plasma }}$ ). For single samples, the PR was the ratio between the WS concentration and the plasma concentration $\left(C_{\mathrm{ws}} / C_{\text {plasma }}\right)$. The significance of the differences between WS and plasma concentrations of AFG, MFG, or CAS was 


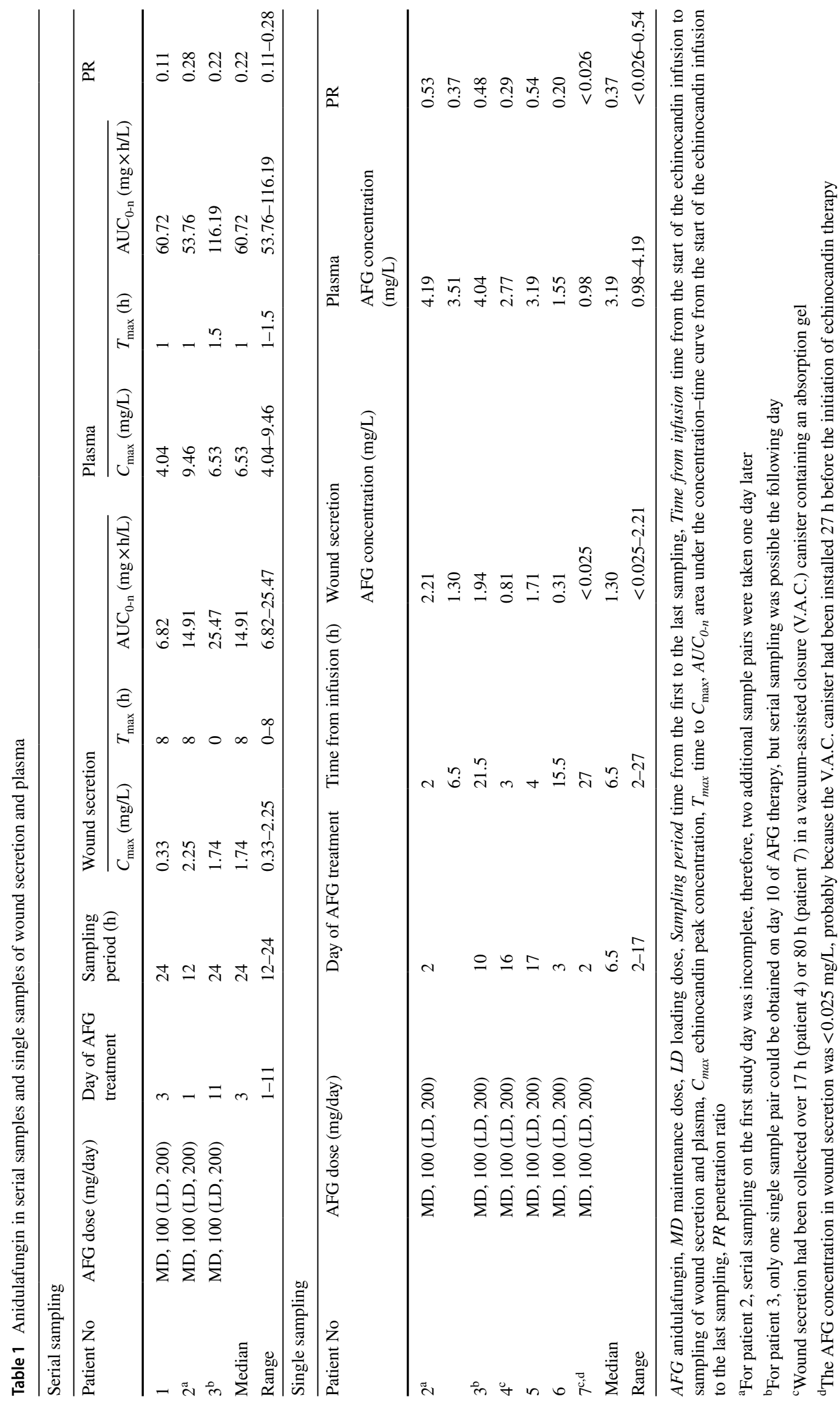




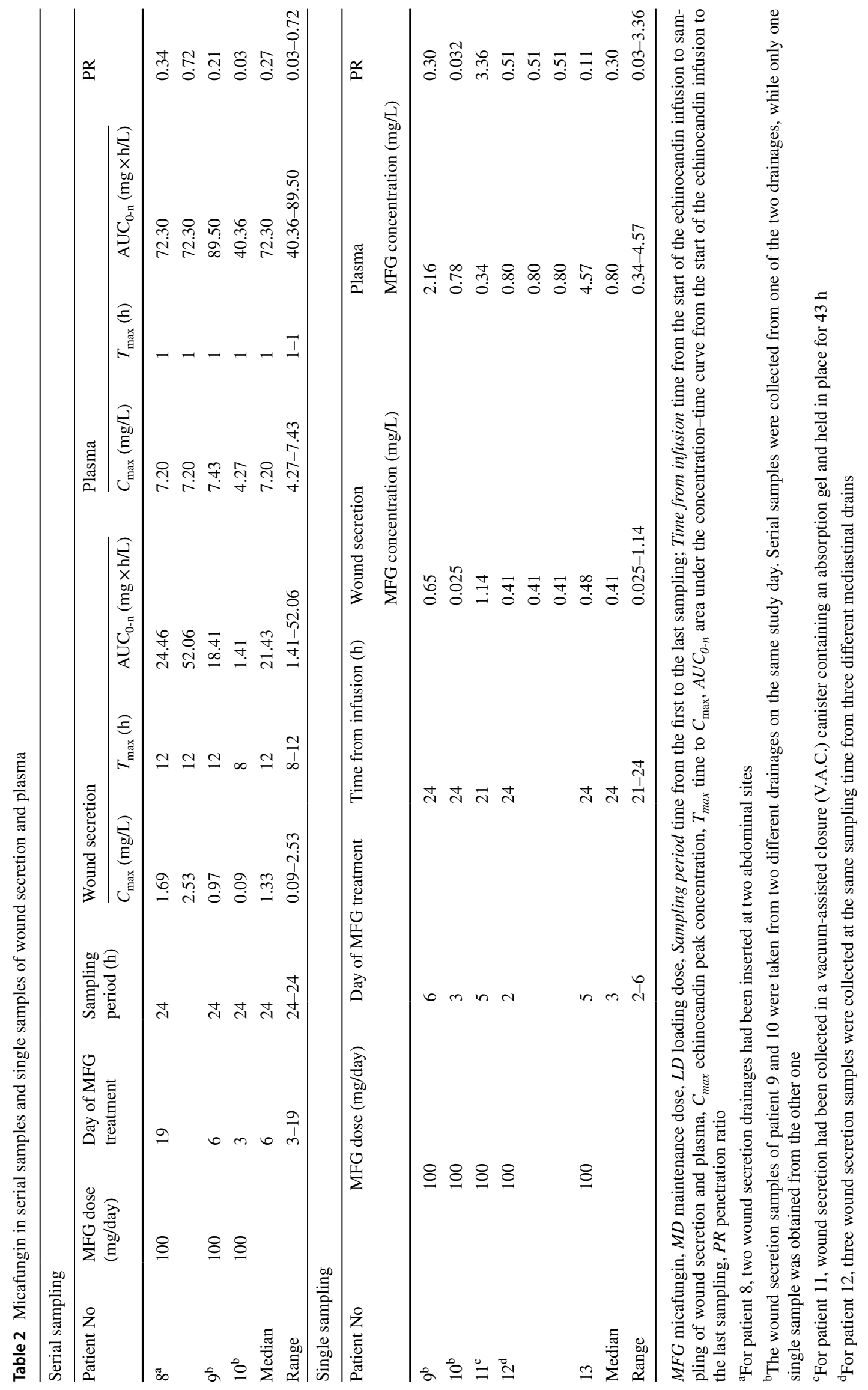


calculated with the Wilcoxon matched-pairs test. The significance of the differences between the concentrations of AFG, MFG, and CAS and between the PRs of the three echinocandins was assessed with the Mann-Whitney $U$ test and Bonferroni correction. PRs calculated from $\mathrm{AUC}_{0-\mathrm{n} \text { ws }} / \mathrm{AUC}$ 0-n plasma and $C_{\mathrm{ws}} / C_{\text {plasma }}$ were considered in equal measure. The IBM SPSS ${ }^{\circledR}$ Statistics software version 26.0 (Armonk, NY, USA) was used for the statistical calculations.

\section{Results}

\section{Study population}

Twenty-one patients were enrolled in this study. Eleven were females. The characteristics of the study patients and the detail of sampling are summarized in Online Resource 2. A total of 70 sample pairs (WS and plasma) were analysed. Serial sample pairs, allowing for calculation of echinocandin pharmacokinetics in WS and in plasma, were obtained from eight patients. Three of these patients were on AFG, three on MFG, and two on CAS. From seventeen patients, single sample pairs were collected at different times from echinocandin infusion (Online Resource 2, Tables 1, 2 and 3). Candida species were isolated from WS of nine study patients (Online Resource 2). Minimal inhibitory concentrations (MICs) of six isolates were determined by E-test ${ }^{\circledR}$ and amounted to $0.002-0.38 \mathrm{mg} / \mathrm{L}$. Four patients presented candidemia. Ten patients were discharged from hospital within 4 months after start of echinocandin therapy, while eleven patients died in ICU or in hospital (Online Resource 2).

\section{Echinocandin concentrations and pharmacokinetics}

In WS, echinocandin concentrations were lower than in plasma $(P$ value $<0.0001)$. The AFG concentrations in WS samples ranged from $<0.025-2.25 \mathrm{mg} / \mathrm{L}$, and the MFG concentrations were $0.025-2.53 \mathrm{mg} / \mathrm{L}$. In the corresponding plasma samples, the AFG concentrations were 0.98-9.46 mg/L, and the MFG concentrations amounted to $0.34-7.43 \mathrm{mg} / \mathrm{L}$. CAS achieved higher concentrations with $0.18-4.04 \mathrm{mg} / \mathrm{L}$ in $\mathrm{WS}$ and $1.84-23.60 \mathrm{mg} / \mathrm{L}$ in plasma $(P$ value $<0.002$ and $<0.05$, respectively). There was no significant difference in PRs between the three echinocandins ( $P$ value $>0.05$, Tables 1,2 and 3 ).

Serial sampling revealed a slower rise and decline of echinocandin concentrations in WS than in plasma. The area under the concentration-time curve over $24 \mathrm{~h}\left(\mathrm{AUC}_{0-24}\right)$ for AFG in WS was 6.82 and $25.47 \mathrm{mg} \times \mathrm{h} / \mathrm{L}$ on day 3 and 11 , respectively, and amounted to 1.41 and $52.06 \mathrm{mg} \times \mathrm{h} / \mathrm{L}$ for MFG on day 3 and 19, respectively. For CAS, an AUC 0-18 of 53.28 and an $\mathrm{AUC}_{0-24}$ of $56.90 \mathrm{mg} \times \mathrm{h} / \mathrm{L}$ were determined (treatment day 3 and 7, respectively). In plasma, the respective AUC values amounted to 53.76-116.19 $\mathrm{mg} \times \mathrm{h} / \mathrm{L}$ for $\mathrm{AFG}, 40.36-89.50 \mathrm{mg} \times \mathrm{h} / \mathrm{L}$ for $\mathrm{MFG}$, and 70.46-277.97 $\mathrm{mg} \times \mathrm{h} / \mathrm{L}$ for CAS (Tables 1, 2 and 3).

The single sample pairs, which were taken on various treatment days and at different time from infusion, yielded highly variable echinocandin concentrations and PRs. The median PR was 0.37 for AFG, 0.30 for MFG, and 0.12 for CAS (differences not significant, see Tables1, 2 and 3).

\section{Discussion}

Yamada et al. reported a MFG concentration of 4.42 (3.90-4.93) $\mathrm{mg} / \mathrm{L}$ with a PR of $0.46(0.40-0.51)$ [median (range)] in WS of a critically ill patient, two to four hours after infusion of a 150-mg dose at steady state [7]. So far, this has been the only report on penetration of an echinocandin into WS. A MFG concentration of $0.38 \mathrm{mg} / \mathrm{L}$ was measured in pancreatic pseudocyst fluid on the seventh day of therapy with $100 \mathrm{mg}$ of MFG daily [9]. In burn eschar, MFG

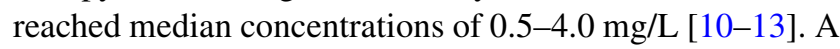
necrotizing fasciitis caused by $C$. albicans following thyroidectomy healed under AFG therapy combined with surgical debridement [1]. Azoles and amphotericin B were also applied for treatment of fungal wound infections [14]. In WS of two patients treated with liposomal amphotericin B ( $5 \mathrm{mg} / \mathrm{kg}$ daily) for 4 and 15 days, respectively, amphotericin B concentrations were between 0.2 and $3.0 \mathrm{mg} / \mathrm{L}$. Voriconazole achieved concentrations of $0.6-2.7 \mathrm{mg} / \mathrm{L}$ in WS of one of the patients [15].

MICs of echinocandins were determined in Candida isolates from wounds of six of our study patients. All the MICs were below the echinocandin concentrations measured in WS of these patients. In-vitro MIC values of echinocandins range from $\leq 0.008$ to $1.0 \mathrm{mg} / \mathrm{L}$ for $C$. albicans and C. glabrata, from 0.03 to $0.25 \mathrm{mg} / \mathrm{L}$ for $C$. krusei, and from $\leq 0.5$ to $2.0 \mathrm{mg} / \mathrm{L}$ for $C$. lusitaniae [16]. The pharmacokinetic-pharmacodynamic target parameter that best correlates with the efficacy of echinocandin treatment is the ratio between the $\mathrm{AUC}_{0-24}$ and the MIC of the pathogen $\left(\mathrm{AUC}_{0-24} / \mathrm{MIC}\right)[17,18]$. Andes et al. reported a fungistatic effect on Candida species for $\mathrm{AUC}_{0-24} / \mathrm{MIC}$ ratios exceeding 2,782 for AFG, 5,299 for MFG, and 748 for CAS [17]. We calculated this ratio when serial samples over $24 \mathrm{~h}$ had been obtained and Candida had been cultivated from a wound. The $\mathrm{AUC}_{0-24} / \mathrm{MIC}$ ratio achieved in patient 3, who had been treated with AFG, and in patient 15 treated with CAS, amounted to 1,592 , and 14,225 , respectively, suggesting an adequate local exposure for patient 15 , but not for patient 3 .

Some limitations of our study must be considered. WS was collected from different anatomical sites via conventional drainage or during V.A.C. therapy, resulting in considerable heterogeneity of WS. The high viscosity of some 


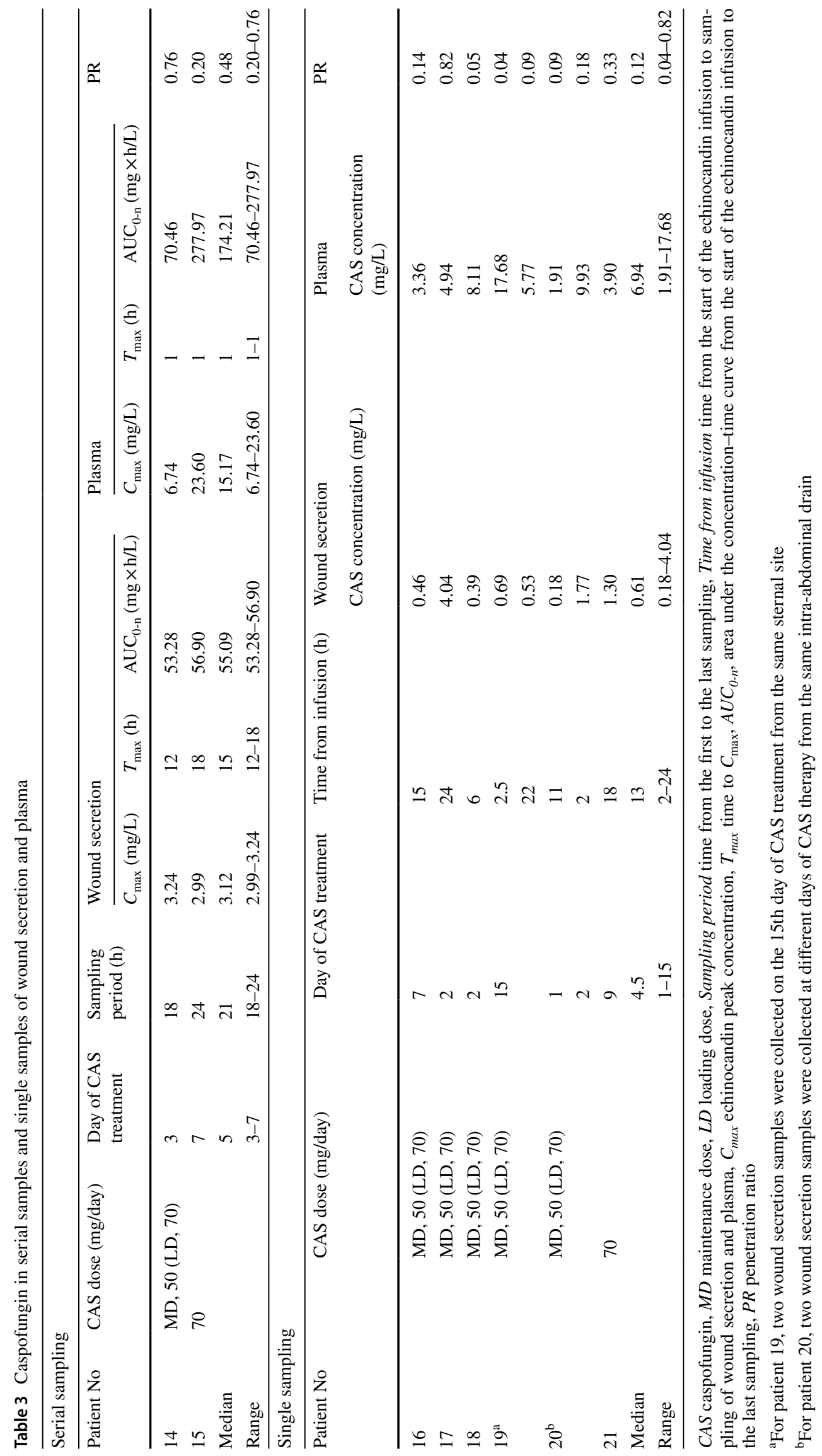


of the WS samples hampered the measurement. Differences in WS composition, particularly in protein and lipid content, might have affected the distribution of echinocandins [19]. The plasma protein binding of CAS amounts to $96 \%$ which is lower than that of AFG (99\%) and MFG (99.9\%) [19]. In the present study, we measured the total drug concentration only, without discrimination between the protein-bound and free echinocandin fraction. The larger free fraction of CAS might favour target site penetration. Accordingly, CAS has achieved higher WS concentration than AFG and MFG. But the PR of CAS was not significantly different from the PRs of AFG and MFG, probably, because also the plasma concentrations of CAS exceeded AFG and MFG plasma levels. In WS, however, the extent of protein binding of echinocandins is unknown, but it might largely affect their antifungal activity. Our study population comprised of critically ill patients presenting with quite different underlying diseases, comorbidities, and constitution. This might have contributed to the high inter-individual variability of echinocandin WS concentrations [20-23]. This variability impedes the interpretation of our findings but largely reflects the situation in critical care medicine. In critically ill patients, highly variable pharmacokinetics of AFG, MFG, and CAS was reported even for serum pharmacokinetics [24]. Furthermore, samples were taken after different treatment duration, and six of our twenty-one study patients (patient 1,2, 6, 7, 12, and 20) had not yet reached steady state. From thirteen patients, we obtained only single sample pairs at different times from echinocandin infusion. Thus, the interval between administration and sampling affected PR because of hysteresis [25]. V.A.C. canisters were in place for up to $80 \mathrm{~h}$ (patient 7, day 2 of AFG therapy). Echinocandin concentrations measured in these samples might, therefore, reflect average WS concentration that was influenced by significant dilution. Chemical degradation of echinocandins cannot be ruled out in WS obtained from V.A.C. canisters. The relevance of in-vitro MIC values for WS is unknown. Thus, WS concentrations exceeding in-vitro MICs do not prove their antifungal efficacy at target-site. Only four of our study patients presented candidemia. Candida was isolated from WS of nine study patients representing probably colonization. Candida was cultivated from both blood and WS of two patients. Our study addressed target-site penetration and pharmacokinetics of echinocandins and was, therefore, not designed and not powered for the assessment of clinical outcome.

Controlled clinical trials on medical treatment of fungal wound infections have not yet been published. The few available data on WS penetration of antifungal drugs as well as the results of the present study suggest a highly variable but limited accessibility of WS. Reliable efficacy of echinocandins applied at standard doses against fungal wound infections can be anticipated only when the causative pathogen is highly susceptible. Thus, timely and thorough surgical debridement has probably a pivotal role in this condition. In addition, high-dose echinocandin treatment, e.g. caspofungin $150 \mathrm{mg}$ daily, could be considered under close monitoring for toxicity [26]. This approach, however, will require evaluation in clinical outcome studies.

\section{Conclusions}

Echinocandin penetration into WS displays a high inter-individual variability. In WS of some of the patients, concentrations may be sub-therapeutic. However, the relevance of sub-therapeutic concentrations is unknown as no correlation has been established between concentration data and clinical outcome. Nevertheless, in the absence of clinical outcome studies, our data do not support the use of echinocandins at standard doses for the treatment of fungal wound infections but underline the pivotal role of surgical debridement.

Supplementary Information The online version contains supplementary material available at https://doi.org/10.1007/s15010-021-01604-x.

Acknowledgements We thank Thomas Nachtigall (Obersöchering, Germany) for donating the HPLC device, and we thank the nurses of the participating ICUs for their support.

Author contributions TG: investigation, methodology, data curation, formal analysis, software, validation, visualization, writing-original draft; RW: investigation, methodology, validation, writing-review and editing; HO: investigation, methodology, data curation, formal analysis, software, validation, writing-review \& editing; JM: methodology, validation; IL: project administration, resources, conceptualization; PS: resources, project administration; TS: resources; KB: investigation, resources, project administration; PE: resources, project administration; TS: data curation, formal analysis, software; AG: resources; HP: investigation; SE: resources, project administration; MA: data curation, writing-review and editing; MJ: resources; RB: conceptualization, data curation, formal analysis, funding acquisition, investigation, methodology, project administration, resources, software, supervision, visualization, writing-review and editing.

Funding Open access funding provided by University of Innsbruck and Medical University of Innsbruck. The study was supported by the Austrian Science Fund (FWF), Grant Number KLI 565-B31.

Code availability Echinocandin pharmacokinetics were calculated using Kinetica 2000® (InnaPhase Corporation, Champs-sur-Marne, France). Statistical analysis was conducted using IBM SPSS $®$ Statistics software version 26.0 (Armonk, NY, USA).

\section{Declarations}

Conflict of interest Peter Schellongowski reports personal fees from Astro-Pharma, Biotest, Novartis, KITE/Gilead, Shire, Pfizer and grants from Astro-Pharma outside the submitted work. Tobias Santner reports personal fees from Roche Diagnostics outside the submitted 
work. Romuald Bellmann reports personal fees from Merck Sharp \& Dohme and Pfizer outside the submitted work.

Ethics approval The study was approved by the local ethics committee (EudraCT no. 2013-005065-38). The study was carried out in accordance with the Declaration of Helsinki and Austrian law.

Consent to participate Written informed consent was obtained from the patients.

Consent for publication All authors read and approved the final manuscript.

Availability of data and material The datasets used and analysed during the current study are available from the corresponding author on request.

Open Access This article is licensed under a Creative Commons Attribution 4.0 International License, which permits use, sharing, adaptation, distribution and reproduction in any medium or format, as long as you give appropriate credit to the original author(s) and the source, provide a link to the Creative Commons licence, and indicate if changes were made. The images or other third party material in this article are included in the article's Creative Commons licence, unless indicated otherwise in a credit line to the material. If material is not included in the article's Creative Commons licence and your intended use is not permitted by statutory regulation or exceeds the permitted use, you will need to obtain permission directly from the copyright holder. To view a copy of this licence, visit http://creativecommons.org/licenses/by/4.0/.

\section{References}

1. Aslier M. Necrotizing fasciitis due to candida infection after thyroid surgery. Turk Arch Otorhinolaryngol. 2020;58(1):56-60. https://doi.org/10.5152/tao.2020.4927.

2. Rodriguez CJ, Tribble DR, Malone DL, Murray CK, Jessie EM, Khan M, et al. Treatment of suspected invasive fungal infection in war wounds. Mil Med. 2018;183(2):142-6. https://doi.org/10. 1093/milmed/usy079.

3. McGraw C, Carrick M, Ekengren F, Berg G, Lieser M, Orlando A, et al. Severe fungal infections following blunt traumatic injuries: a 5-year multicenter descriptive study. Injury. 2019;50(12):2234-9. https://doi.org/10.1016/j.injury.2019.10.027.

4. Arıkan AA, Omay O, Kanko M, Horuz E, Yağlı G, Kağan EY, et al. Treatment of Candida sternal infection following cardiac surgery - a review of literature. Infect Dis (Lond). 2019;51(1):1-11. https://doi.org/10.1080/23744235.2018.1518583.

5. Palackic A, Popp D, Tapking C, Houschyar KS, Branski LK. Fungal infections in burn patients. Surg Infect (Larchmt). 2020. https://doi.org/10.1089/sur.2020.299.

6. Pappas PG, Kauffman CA, Andes DR, Clancy CJ, Marr KA, Ostrosky-Zeichner L, et al. Clinical practice guideline for the management of Candidiasis: 2016 update by the infectious diseases society of America. Clin Infect Dis. 2016;62(4):e1-50. https://doi.org/10.1093/cid/civ933.

7. Yamada N, Kumada K, Kishino S, Mochizuki N, Ohno K, Ogura $\mathrm{S}$. Distribution of micafungin in the tissue fluids of patients with invasive fungal infections. J Infect Chemother. 2011;17(5):731-4. https://doi.org/10.1007/s10156-011-0240-3.

8. Welte R, Oberacher H, Schwärzler B, Joannidis M, Bellmann R. Quantification of anidulafungin and micafungin in human body fluids by high performance-liquid chromatography with UV-detection. J Chromatogr B Analyt Technol Biomed Life
Sci. 2020;1139:121937. https://doi.org/10.1016/j.jchromb.2019. 121937.

9. Lat A, Thompson GR 3rd, Rinaldi MG, Dorsey SA, Pennick G, Lewis JS 2nd. Micafungin concentrations from brain tissue and pancreatic pseudocyst fluid. Antimicrob Agents Chemother. 2010;54(2):943-4. https://doi.org/10.1128/AAC.01294-09.

10. García-de-Lorenzo A, Luque S, Grau S, Agrifoglio A, Cachafeiro L, Herrero E, et al. Comparative population plasma and tissue pharmacokinetics of micafungin in critically Ill patients with severe burn injuries and patients with complicated intra-abdominal infection. Antimicrob Agents Chemother. 2016;60(10):5914 21. https://doi.org/10.1128/AAC.00727-16.

11. Asensio MJ, Sánchez M, Galván B, Herrero E, Cachafeiro L, Agrifoglio A, et al. Micafungin at a standard dosage of $100 \mathrm{mg} /$ day achieves adequate plasma exposure in critically ill patients with severe burn injuries. Intensive Care Med. 2015;41(2):371-2. https://doi.org/10.1007/s00134-014-3586-z.

12. Sasaki J, Yamanouchi S, Kudo D, Endo T, Nomura R, Takuma K, et al. Micafungin concentrations in the plasma and burn eschar of severely burned patients. Antimicrob Agents Chemother. 2012;56(2):1113-5. https://doi.org/10.1128/AAC.05381-11.

13. Sasaki J, Yamanouchi S, Sato Y, Abe S, Shinozawa Y, Kishino $S$, et al. Penetration of micafungin into the burn eschar in patients with severe burns. Eur J Drug Metab Pharmacokinet. 2014;39(2):93-7. https://doi.org/10.1007/s13318-013-0146-9.

14. Malani PN, McNeil SA, Bradley SF, Kauffman CA. Candida albicans sternal wound infections: a chronic and recurrent complication of median sternotomy. Clin Infect Dis. 2002;35(11):1316-20. https://doi.org/10.1086/344192.

15. Akers KS, Rowan MP, Niece KL, Graybill JC, Mende K, Chung $\mathrm{KK}$, et al. Antifungal wound penetration of amphotericin and voriconazole in combat-related injuries: case report. BMC Infect Dis. 2015;15:184. https://doi.org/10.1186/s12879-015-0918-8.

16. Pfaller MA, Messer SA, Rhomberg PR, Castanheira M. CD101, a long-acting echinocandin, and comparator antifungal agents tested against a global collection of invasive fungal isolates in the SENTRY 2015 antifungal surveillance program. Int J Antimicrob Agents. 2017;50(3):352-8. https://doi.org/10.1016/j.ijantimicag. 2017.03.028.

17. Andes D, Diekema DJ, Pfaller MA, Bohrmuller J, Marchillo K, Lepak A. In vivo comparison of the pharmacodynamic targets for echinocandin drugs against Candida species. Antimicrob Agents Chemother. 2010;54(6):2497-506. https://doi.org/10.1128/AAC. 01584-09.

18. Andes D, Ambrose PG, Hammel JP, Van Wart SA, Iyer V, Reynolds DK, et al. Use of pharmacokinetic-pharmacodynamic analyses to optimize therapy with the systemic antifungal micafungin for invasive candidiasis or candidemia. Antimicrob Agents Chemother. 2011;55(5):2113-21. https://doi.org/10.1128/AAC. 01430-10.

19. Bellmann R, Smuszkiewicz P. Pharmacokinetics of antifungal drugs: practical implications for optimized treatment of patients. Infection. 2017;45(6):737-79. https://doi.org/10.1007/ s15010-017-1042-z.

20. Nguyen TH, Hoppe-Tichy T, Geiss HK, Rastall AC, Swoboda $\mathrm{S}$, Schmidt J, et al. Factors influencing caspofungin plasma concentrations in patients of a surgical intensive care unit. J Antimicrob Chemother. 2007;60(1):100-6. https://doi.org/10.1093/jac/ dkm125.

21. Liu P, Ruhnke M, Meersseman W, Paiva JA, Kantecki M, Damle B. Pharmacokinetics of anidulafungin in critically ill patients with candidemia/invasive candidiasis. Antimicrob Agents Chemother. 2013;57(4):1672-6. https://doi.org/10.1128/AAC.02139-12.

22. Smith BS, Yogaratnam D, Levasseur-Franklin KE, Forni A, Fong J. Introduction to drug pharmacokinetics in the critically 
ill patient. Chest. 2012;141(5):1327-36. https://doi.org/10.1378/ chest.11-1396.

23. Lempers VJ, Schouten JA, Hunfeld NG, Colbers A, van Leeuwen HJ, Burger DM, et al. Altered micafungin pharmacokinetics in intensive care unit patients. Antimicrob Agents Chemother. 2015;59(8):4403-9. https://doi.org/10.1128/AAC.00623-15.

24. Mainas E, Apostolopoulou O, Siopi M, Apostolidi S, Neroutsos E, Mirfendereski H, et al. Comparative pharmacokinetics of the three echinocandins in ICU patients. J Antimicrob Chemother. 2020;75(10):2969-76. https://doi.org/10.1093/jac/dkaa265.
25. Felton T, Troke PF, Hope WW. Tissue penetration of antifungal agents. Clin Microbiol Rev. 2014;27(1):68-88. https://doi.org/10. 1128/CMR.00046-13.

26. Betts RF, Nucci M, Talwar D, Gareca M, Queiroz-Telles F, Bedimo RJ, et al. A Multicenter, double-blind trial of a high-dose caspofungin treatment regimen versus a standard caspofungin treatment regimen for adult patients with invasive candidiasis. Clin Infect Dis. 2009;48(12):1676-84. https://doi.org/10.1086/ 598933. 